\section{Cover illustration: Eyes in the back of your head}

The American woodcock (Scolopax minor, although the older name, Philohela minor from the Greek meaning "loving marsh" seems much more appropriate) is a shy upland game bird, which is a member of the sandpiper family. This species and its closely related European counterpart, Scolopax rusticola, has quit the water's edge for marsh or even dense woodlands with a moist forest floor. The European woodcock is a similar, but larger, bird with similar habits, coloration, and ecology. The migratory and secretive American woodcock, now in decline, nests primarily in the northern portion of its range in North America, especially in young forests with scattered openings having swamplike moist floors. The female is larger, in general, but usually this cannot be determined in the field. The distinctive long tapered bill is used to forage by probing for earthworms in damp soil, soft substrates, or in alder thickets with associated clearings and boggy fens. The bird is difficult, if not impossible, to see because of cryptic coloration, as can be determined by the photograph on this month's cover. The leaf litter coloration of its feathers allows the bird to settle on the forest floor as a means of camouflage and is is rarely seen unless flushed out. The flight is usually a brief irregular halting one through the surrounding trees until it disappears again upon landing. The bird is

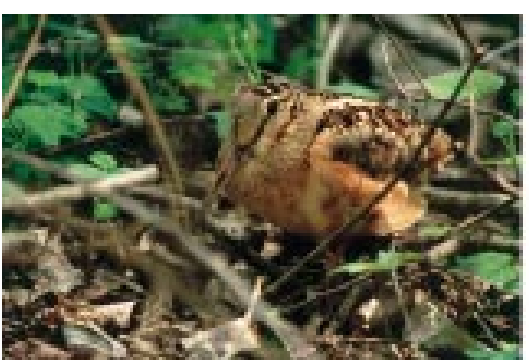

The woodcock probably doesn't have particularly good acuity, but it does have an extraordinary ocular feature probably shared by few other land vertebrates. Although extraocular motility is probably limited, these birds have a most unusual visual field. The large eyes are set high on the head as can be seen from the photograph. Living on the ground, the bird risks death by ground or aerial predators and must protect itself with camouflage and stealth, and yet it does not really need its vision for feeding. Acuity, then, is not at a premium, but protection against predation is, and that requires movement detection and a large visual field. This bird has both.

The visual field is shown in the sketch on the cover. It is extraordinary, and has been documented (Martin GR, Visual fields in woodcocks Scolopax rusticola (Scolopaci-dae; Charadriiformes). F Comp Physiol A 1994; 174:787-93). There is a $5-12^{\circ}$ binocular field in the frontodorsal quadrant beginusually active at dusk or into the night and may fly at tree top level. When they migrate, they may travel in loose groups of hundreds or even thousands.

Perhaps, if lucky, you may see the male perform the spectacular courtship flight to advertise his presence to a female. This flight, usually performed initially on wintering grounds in late January, will continue on so called "singing sites" in the best nesting sites in northern North America throughout the season or at least into summer. The flight or nuptial dance is renowned among naturalists because of its intricate beauty and complexity. The male begins by leaping from the ground, rising in ever widening circles to about 300-400 feet overhead. He will hover overhead briefly as he sings a series of chirps. Then, he drops, still chirping in a series of reverse switchbacks similar to a falling maple leaf zigzaggimg across an air current. He alights on the ground, sings a different note, and walks stiffly to the waiting female, whereupon they copulate. Males will often mate with many females. These crepuscular or nocturnal birds feed mostly at dusk or during the night, but rarely during the day. The bird will stamp its foot on the ground with quick sharp blows to stir the earthworms into movement before probing the earth, and it can eat its weight or more in earthworms within 24 hours. It is believed that the woodcock can feel or smell the earthworm as it probes the loose, moist dirt. The bill tip may feel the vibrations of an earthworm's movement in the soil, but whatever the mechanism, the bird does not use sight to see the earthworm as we shall learn. If necessary, it will also eat slugs, other invertebrates, and even salamanders and small frogs. The nest is a slight hollow, lined with forest litter, often in underbrush. The female incubates and the urge to remain on the nest is so strong that the female can be stroked while sitting on eggs, if you can find the bird that is. If she has hatchlings, she will act like many other sandpipers with an "injured bird" feint to draw the predator away. Once the predator has followed her a sufficient distance from the nest, she will regain her wings and depart unhurt to return to the nest. The hatchlings mature rapidly and are fully grown at 28 days.

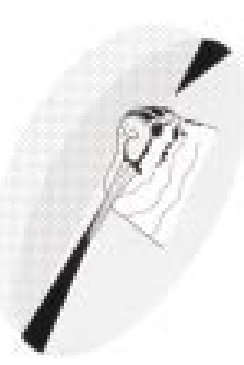

ning a few degrees above the upper mandible as seen shaded in black in the sketch. There is, in fact, a blind spot at the tip of the bill because the bill is directed downwards. That means the bird cannot see the tip of its bill and so could not use its sight for direct foraging, but it doesn't need to. There is a simultaneous $5^{\circ}$ binocular visual field behind the head although others have predicted a larger field posteriorly (Walls, 1942) also seen in black in the drawing. This bird has a binocular field with presumed stereopsis simultaneously in front along the vertical sagittal meridian and directly and behind the head, albeit a rather small binocular field. There's more. There is a monocular field that completes the $360^{\circ}$ field connecting the edges of the binocular fields. This cyclopean portion of the field is illustrated with grey shading in the sketch on the cover. The visual field also extends in a hemispherical fashion above the head, creating a $360^{\circ}$ hemispherical field which even extends $5-10^{\circ}$ below the horizontal. The vertical field is illustrated by the dotted area on the sketch. Conceptually then, this bird can sit on its nest and have the entire visual space around it encompassed, save for the bilateral blind spots of the pecten (the intraocular nutritive organ, homologous to the central retinal artery to be discussed in a future cover) in each cyclopean field laterally slightly above the horizontal. Evolutionarily, this makes complete sense. The species does not need vision for feeding, given the sensory capabilities of the mandible, but living as it does on the forest floor, aerial and terrestrial predation is a risk and it is essential to achieve protection and any such mechanism would be conserved. This must be the case since this bird has been documented since at least the Pleistocene era. Ecologically, this bird has gone as far as it needs to go for predator protection because acuity is not needed or even possible for feeding for this animal. While some fish have very than the American woodcock, this is almost certainly the largest visual field in a terrestrial vertebrate.

This bird would have been an all knowing primary school teacher with eyes in the back of her head!-Ivan $R$ Schwab, MD, UC Davis Department of Ophthalmology, 4860 Y Street, Ste 2400, Sacramento, CA 95817, USA (irschwab@ucdavis.edu)

Thanks to Bruce Hallett for the photograph of the American woodcock. Thanks to Kariann Reno for the study specimens. large visual fields and some may even have larger visual fields 